\title{
Stability and Distributed Control of Degenerate Diffusion Equations
}

\author{
Andrea Cristofaro, Roberto Giambò and Fabio Giannoni
}

\section{Introduction}

Partial differential equations and distributed parameter systems have attracted the attention of the control community (see [14], [18], [7], [3] among several others) since many plant models are described by infinite-dimensional systems and hence involve PDEs or systems of PDEs: examples can be found in robotics (haptic controllers and flexible manipulators), in industrial processes (manufacturing, reactors and heat transfer plants) as well as in biomedical applications (tissue engineering and teleoperated surgery).

A large effort has been made to develop classical control tools and establish basic results for such infinite-dimensional dynamical systems, e.g. stability [8], [6], constrained control [25], optimal control [28], [33], robust control [31], [29], [10] and observer design [32]. However, the majority of such results pertains classes of linear or quasilinear parabolic and hyperbolic equations. Tackling nonlinear equations is usually much harder, and requires to carefully address some delicate points, which are instead straightforward in the linear case. Among nonlinear problems, equations involving degenerate operators, such as the $p$-Laplace operator, are particularly challenging. The $p$-Laplace equation, which is the natural extension of the classical Laplace equation onto the space $L^{p}$, and the corresponding solutions, called $p$-harmonic functions, are used for modeling physical phenomena arising from glaciology, radiation of heat, or plastic moulding [22]. Existence, boundedness, estimates and regularity of solutions have been largely investigated over the last two decades, see for instance $[24,17,4,1,30,12,5,9]$. The classical theory of parabolic equations is not sufficient to guarantee that the time-derivative of the solution of the parabolic $p$-Laplace equation does exists as a function, even though the space-derivatives are Hölder continuous functions. A regularity theory for the time-derivative of the solution has been developed only in recent years $[23,13]$.

Asymptotic convergence of the solution of the $p$-parabolic equation to the stationary one has proved in [21] in the case of time-independent data, while largetime geometric properties of solutions have been exploited in [20]. Interesting connections with stability are also provided by the theory of principal eigenvalues $[16,19]$. However from a control theoretic perspective, to the best of authors' knowledge, no significant result is available yet. As a first step to- 
wards that goal, the Lyapunov stability of the stationary solution has been investigated, this complementing the result of [21] where convergence to zero is proved using parabolic estimates. In particular, the $p$-parabolic equations is shown to be asymptotically stable in $L^{2}$ as well as in $L^{p^{*}}$, where $p^{*}$ is the socalled Sobolev conjugate [2]. Moreover, the presence of lower order terms with a "good" growth has a regularizing effect on the solution and yields exponential stability also. The proposed results extend straightforwardly to a general class of degenerate parabolic problems, whose inspiration model is the parabolic $p$-Laplace equation. Having established these basic stability conditions, which were preliminary presented in [11] and reported here for the sake of completeness, two control problems have been considered for the parabolic $p$-Laplace equation controlled via a distributed input. In particular, given a desired reference profile, a finite-time reference tracking control law and quadratic optimal controller are proposed. In the latter case, the synthesis of the optimal controller is based on the solution of an adjoint equation. A simulation study has been performed to illustrate with numerical examples the different convergence properties of the considered class of equations.

\section{Parabolic $p$-Laplace equation}

The $p$-Laplace operator $\Delta_{p}$ is a generalization of the classical Laplace operator, and for $p \in[1, \infty)$ is defined as

$$
\Delta_{p} v:=\operatorname{div}\left(|\nabla v|^{p-2} \nabla v\right)
$$

where $^{1} v: \mathbb{R}^{n} \rightarrow \mathbb{R}$ is a scalar function with enough regularity. Clearly, such operator is singular for $1 \leq p<2$ and degenerate for $p>2$, i.e. the modulus of ellipticity vanishes for $|\nabla v|=0$. The associated parabolic equation is defined in a natural way as

$$
u_{t}=\operatorname{div}\left(|\nabla u|^{p-2} \nabla u\right)
$$

In the following, we will refer to (1) as the parabolic p-Laplace equation or, shortly, as the p-parabolic equation.

We begin our analysis focusing on the Cauchy-Dirichlet problem for (1), and in particular we will deal with

$$
\left\{\begin{array}{l}
u_{t}=\operatorname{div}\left(|\nabla u|^{p-2} \nabla u\right)+f \\
u(t, x)=0 \quad t \in[0, \infty), x \in \partial \Omega \\
u(0, x)=u_{0}(x) \quad x \in \Omega
\end{array}\right.
$$

where $\Omega \subset \mathbb{R}^{n}, n \geq 2$, is a bounded domain with a smooth boundary, and $u_{0}, f$ are prescribed data whose regularity will be specified later on. Given a finite time horizon $[0, T]$, a weak solution of (2) on the cylinder $Q_{T}:=[0, T] \times \Omega$ is a

\footnotetext{
${ }^{1}$ We recall that, for a given vector field $F(x)=\left[F_{1}(x), \cdots, F_{n}(x)\right]$, the divergence operator is defined by $\operatorname{div} F(x)=\partial_{x_{1}} F_{1}(x)+\partial_{x_{2}} F_{2}(x)+\cdots+\partial_{x_{n}} F_{n}(x)$.
} 
function $u$ in a local parabolic Sobolev space that satisfies the identity

$$
\int_{0}^{T} \int_{\Omega}\left(-u \varphi_{t}+|\nabla u|^{p-2} \nabla u^{T} \nabla \varphi\right) d x d t=\int_{0}^{T} \int_{\Omega} f \varphi d x d t
$$

for any function $\varphi \in C_{0}^{1}\left(\bar{Q}_{T}\right)$. The definition can be readily extended to the infinite cylinder $[0, \infty) \times \Omega$ by considering test functions $\varphi$ with compact support in $[0, \infty] \times \bar{\Omega}$.

\subsection{Existence and regularity of solutions}

Assume $^{2} p \geq 2$ and introduce the functional space

$$
V^{p}(0, T ; \Omega)=C\left(0, T ; L^{2}(\Omega)\right) \cap L^{p}\left(0, T ; W_{0}^{1, p}(\Omega)\right) .
$$

In formulae, a function $v(t, x)$ belongs to $V^{p}(0, T ; \Omega)$ if the mapping $t \mapsto$ $\|v(t, \cdot)\|_{2}$ is continuous, and the integral $\int_{0}^{T} \int_{\Omega}|\nabla v(t, x)|^{p} d x d t$ is finite. Following $[17,12]$, for any fixed $T$ the existence of a local bounded weak solution $u \in V^{p}(0, T ; \Omega)$ can be proved under the assumptions:

A1) The function $f=f(t, x)$ verifies $f(\cdot, x) \in L^{\infty}(0, \infty)$ for any $x \in \Omega$ and $f(t, \cdot) \in L^{p^{\prime}}(\Omega)$ for any $t \in[0, \infty)$, where the number $p^{\prime}$ is the Lebesgue conjugate of $p$, i.e. $1 / p+1 / p^{\prime}=1$.

A2) The function $u_{0}(x) \in W_{0}^{1, p}(\Omega)$.

Without loss of generality, it will be therefore assumed that for any $T>0$ a solution $u \in L^{\infty}\left(Q_{T}\right)$ exists. In fact, solutions can be proved to be Hölder continuous. Moreover, unicity of the weak solutions is provided by the following useful algebraic inequality:

$$
\left(|\zeta|^{p-2} \zeta-|z|^{p-2} z\right) \cdot(\zeta-z) \geq 2^{1-p}|\zeta-z|^{p}
$$

for any $\zeta, z \in \mathbb{R}^{n}$. Putting all the pieces together, we can infer that a unique solution $u \in V_{l o c}^{p}(0, \infty ; \Omega) \cap L_{l o c}^{\infty}\left(Q_{\infty}\right)$ exists, where $Q_{\infty}:=[0, \infty) \times \Omega$.

Remark 2.1 Thanks to the regularity of solutions, the equality (3) extends to any function $\varphi \in W_{0}^{1, p}\left(Q_{T}\right)$.

\section{Lyapunov stability in $L^{2}$}

Let us begin our analysis by considering the homogeneous problem, i.e. $f \equiv$ 0 . The zero function $u^{*} \equiv 0$ is clearly a weak (stationary) solution of the Cauchy-Dirichlet problem $(2)$ when $u_{0}(x)=0$. Let us prove that such stationary

\footnotetext{
${ }^{2}$ We notice that the given existence and regularity results still hold in the larger range $p \geq 2 n /(n-1)$. However, in this case the inequality (4) is slightly different [17]. For $p<$ $2 n /(n-1)$ the local boundedness of solutions is instead no longer guaranteed.
} 
solution is an asymptotically stable equilibrium for the homogeneous $p$-parabolic equation. To this end, let us consider the Lyapunov function candidate

$$
V(t):=\frac{1}{2} \int_{\Omega}|u(t, x)|^{2} d x .
$$

Recall that for a domain $\Omega$ with $|\Omega|<\infty$ the Lebesgue spaces are encapsulated with $L^{q}(\Omega) \subset L^{r}(\Omega)$ for $r<q$. Since $p \geq 2$, the solution $u(t, x)$ is in $L^{p}(\Omega) \subseteq$ $L^{2}(\Omega)$ for any fixed $t$, and hence the function $V(t)$ is well-defined. The aim is to evaluate the derivative of $V(t)$ along the solution; however, one has to be careful while performing such operation, as in principle $u_{t}$ only exists as a distribution and thus differentiation under the integral sign might be not allowed. On the other hand, some recent results pertaining the regularity of time derivatives may be invoked [13, 23].

Proposition 3.1 Let $u \in V_{l o c}^{p}(0, \infty ; \Omega) \cap L^{\infty}\left(Q_{\infty}\right)$ be the weak solution of (2); assume in addition that assumptions $\mathrm{A} 1$ and $\mathrm{A} 2$ hold. Then the time derivative $u_{t}$ satisfies:

$$
\begin{aligned}
& \text { i) } u_{t} \in L_{l o c}^{2}\left(0, \infty ; L_{l o c}^{q}(\Omega)\right) \forall q \in\left[1, \frac{2 n}{n-\frac{1}{2}}\right] \text {; } \\
& \text { ii) } u_{t} \in L_{l o c}^{\infty}\left(0, \infty ; L^{2}(\Omega)\right) .
\end{aligned}
$$

Thanks to the latter result, $u_{t}$ does exist as a function, and verifies the local estimate

$$
\left\|u_{t}(t, \cdot)\right\|_{2} \leq M \quad \forall t \in\left[t_{0}-\epsilon, t_{0}+\epsilon\right]
$$

where $M>0$ only depends on $t_{0}$ and $\epsilon$. Indeed the derivative has even more regularity, as established in the following claim, whose proof is given in Appendix B.

Claim 3.1 Let $\eta>0$ and set

$$
Q_{\eta, \infty}:=(\eta, \infty) \times \Omega .
$$

The solution $u(t, x)$ is differentiable almost everywhere in $Q_{\eta, \infty}$ for any $\eta>0$, and $u_{t}(t, x)$ equals the strong derivative a.e. in $Q_{\eta, \infty}$.

In view of such property, and based on condition ii) of Proposition 3.1, it is reasonable to introduce the following class of functions and, accordingly, make an assumption on the initial datum $u_{0}(x)$.

Definition 3.1 For $p \geq 1$ the class of $L^{p}$-pointwise bounded functions $B\left(a, b ; L^{p}(\Omega)\right)$ is defined as

$$
\begin{gathered}
B\left(a, b ; L^{p}(\Omega)\right):=\quad\left\{f(t, x):|f(t, x)| \leq g_{f}(x) \in L^{p}(\Omega)\right. \\
\forall \text { a.e. } x \in \Omega, \forall t \in(a, b)\}
\end{gathered}
$$


Remark 3.1 It is worth noticing that the following inclusion holds

$$
B\left(a, b ; L^{p}(\Omega)\right) \supset L^{\infty}\left(a, b ; L^{p}(\Omega)\right) .
$$

Let us introduce the following set of initial conditions:

$$
\mathcal{B}_{0}:=\left\{u_{0}(x) \in W_{0}^{1, p}: \begin{array}{c}
\text { the solution } u(t, x) \text { is such that } \\
u_{t}(t, x) \in B\left(0, \infty ; L^{1}(\Omega)\right)
\end{array}\right\}
$$

Recalling that $u(t, x)$ is bounded and differentiable a.e., the assumption $u_{0}(x) \in$ $\mathcal{B}_{0}$ yields the differentiability of $V(t)$ with

$$
\dot{V}(t)=\int_{\Omega} u(t, x) u_{t}(t, x) d x .
$$

Due to this nice property, the following stability result can be established.

Theorem 3.1 Assume $p \geq 2$ and $f \equiv 0$. The stationary solution $u^{*}$ is an $L^{2}$-asymptotically stable equilibrium with a region of attraction $\mathcal{R}_{u^{*}} \supseteq \mathcal{B}_{0}$, i.e. $V(t)$ satisfies a Lyapunov inequality of the type

$$
\dot{V}(t) \leq-c[V(t)]^{\frac{p}{2}}, \quad c>0
$$

for any initial condition $u_{0}(x) \in \mathcal{B}_{0}$.

Proof. As mentioned, the derivative of $V(t)$ can be written as in (6). On the other hand, thanks to Remark 2.1, the solution $u$ can be used indeed as a test function, and hence (by implicitly applying the divergence lemma and canceling out the boundary terms) one has

$$
\dot{V}(t)=-\int_{\Omega}|\nabla u(t, x)|^{p} d x \leq-C_{p} \int_{\Omega}|u(t, x)|^{p} d x,
$$

with $C_{p}>0$, where the last estimate follows from Poincaré's inequality. Finally, using Jensen's inequality (see Appendix A), one gets

$$
\dot{V}(t) \leq-C_{p} \int_{\Omega}|u(t, x)|^{p} d x \leq-c\left(\frac{1}{2} \int_{\Omega}|u(t, x)|^{2}\right)^{\frac{p}{2}}
$$

where $c=2^{\frac{p}{2}}|\Omega|^{1-\frac{p}{2}} C_{p}$. Now, by a standard comparison argument [27], the latter chain of inequalities implies asymptotic stability. Indeed, one has

$$
\frac{1}{2}\|u(t, \cdot)\|_{2}^{2}=V(t) \leq\left(\frac{1}{V(0)^{1-\frac{p}{2}}+c\left(\frac{p}{2}-1\right) t}\right)^{\frac{2}{p-2}}
$$

where $V(0)=\frac{1}{2}\left\|u_{0}(x)\right\|_{2}^{2}$.

Let us investigate the problem further, and work towards obtaining a more gen- 
eral stability result. To this end, let us consider now problem (2) with a datum $f$ satisfying assumption $\mathrm{A} 1$ and independent of $t$, i.e. $f(t, x)=f(x)$. Accordingly, let us denote by $u_{f}^{*}$ the solution of the corresponding elliptic problem or, equivalently, the stationary solution of (2), that is

$$
-\operatorname{div}\left(\left|\nabla u_{f}^{*}\right|^{p-2} \nabla u_{f}^{*}\right)=f
$$

Focusing on such stationary solution, a natural question arises: how is $u_{f}^{*}$ related to the solution of the original $p$-parabolic equation? It has been proved in [21] that, under very mild conditions (e.g. even irregular data), the solution $u(t, x)$ converges to $u_{f}^{*}(x)$ in $L^{1}$ as $t \rightarrow \infty$. We will prove that a stronger property holds under assumptions A1-A2, namely $u_{f}^{*}$ is an asymptotically stable equilibrium in $L^{2}$.

In this regard, consider the modified Lyapunov function candidate

$$
W(t)=\frac{1}{2} \int_{\Omega}\left|u(t, x)-u_{f}^{*}(t, x)\right|^{2} d x .
$$

The following statement constitutes the main stability result of the paper, and generalizes the assessment of Theorem 3.1.

Theorem 3.2 Assume $p \geq 2$ and let $f(t, x)=f(x)$ be such that $\mathrm{A} 1$ is fulfilled. The stationary solution $u_{f}^{*}$ is an $L^{2}$-asymptotically stable equilibrium with a region of attraction $\mathcal{R}_{u_{f}^{*}} \supseteq \mathcal{B}_{0}$, i.e. the function $W(t)$ satisfies a Lyapunov inequality of the type

$$
\dot{W}(t) \leq-c[W(t)]^{\frac{p}{2}}, \quad c>0
$$

for any initial condition $u_{0}(x) \in \mathcal{B}_{0}$.

Proof. Proceeding as in the proof of Theorem 3.1, and using the regularity of the time derivative $u_{t}-u_{f, t}^{*}$, with a slight abuse of notation one has

$$
\begin{aligned}
\dot{W}(t) & =\int_{\Omega}\left(u-u_{f}^{*}\right)\left(u_{t}-u_{f, t}^{*}\right) d x \\
& =\int_{\Omega}\left(u-u_{f}^{*}\right)\left(\Delta_{p} u-\Delta_{p} u_{f}^{*}\right) d x \\
& =\underbrace{\int_{\partial \Omega}\left\langle\left(u-u_{f}^{*}\right)\left(|\nabla u|^{p-2} \nabla u-\left|\nabla u_{f}^{*}\right|^{p-2} \nabla u_{f}^{*}\right), \mathbf{n}\right\rangle d \sigma}_{=0} \\
& -\int_{\Omega}\left(\nabla u-\nabla u_{f}^{*}\right) \cdot\left(|\nabla u|^{p-2} \nabla u-\left|\nabla u_{f}^{*}\right|^{p-2} \nabla u_{f}^{*}\right) d x
\end{aligned}
$$

where the dependency on $(t, x)$ has been omitted and $\mathbf{n}$ stands for the outer normal to $\partial \Omega$. Applying inequality (4) yields

$$
\dot{W}(t) \leq-2^{1-p} \int_{\Omega}\left|\nabla u-\nabla u_{f}^{*}\right|^{p} d x,
$$

and hence, mimicking the steps of the proof of Theorem 3.1, it is straightforward to attain the desired estimate $(9)$ with $c=(2|\Omega|)^{1-\frac{p}{2}} C_{p}$. 


\section{Stability in $L^{p^{*}}$, lower-order terms and gener- alizations.}

For the sake of simplicity we limit to consider the homogeneous case $f \equiv 0$ only: the extension to the general case is straightforward.

\subsection{Lyapunov stability in $L^{p^{*}}$}

A stronger stability result can be proved indeed under the same conditions. Let $2 \leq p<n$ and consider the Lyapunov function candidate

$$
V^{*}(t):=\frac{1}{p^{*}} \int_{\Omega}|u(t, x)|^{p^{*}} d x
$$

where $p^{*}$ is the Sobolev conjugate. Adapting the steps of the proof of Theorem 1 , one gets the inequality

$$
\dot{V}^{*}(t) \leq-\int_{\Omega}|u|^{p^{*}-1}|\nabla u|^{p} d x
$$

Since $u(t, x) \in L^{\infty}\left(Q_{\infty}\right)$ by construction, the function $v(t, x)=\frac{1}{\gamma}|u(t, x)|^{\gamma} \in$ $W_{0}^{1, p}$ for any $\gamma \geq 1$, with

$$
|\nabla v|^{p}=|u|^{p(\gamma-1)}|\nabla u|^{p}
$$

Let us denote by $\gamma^{*}$ the solution to the algebraic equation

$$
p\left(\gamma^{*}-1\right)=p^{*}-1,
$$

that is $\gamma^{*}=1+\frac{p^{*}}{p}-\frac{1}{p} \geq 1$. Applying the Gagliardo-Nirenberg-Sobolev inequality, one gets

$$
\begin{aligned}
\dot{V}^{*}(t) & \leq-\int_{\Omega}|u|^{p^{*}-1}|\nabla u|^{p} d x \\
& \leq-\left(\frac{S_{p}}{\gamma^{*}}\right)^{p}\left(\int_{\Omega}|u|^{\gamma^{*} p^{*}} d x\right)^{\frac{p}{p^{*}}}
\end{aligned}
$$

where $S_{p}$ is the Sobolev constant. Finally, by Jensen's inequality, the integral in the latter term can be bounded as

$$
-\left(\int_{\Omega}|u|^{\gamma^{*} p^{*}} d x\right)^{\frac{p}{p^{*}}} \leq-|\Omega|^{1-\gamma^{*}}\left(\int_{\Omega}|u|^{p^{*}} d x\right)^{\frac{\gamma^{*} p}{p^{*}}}
$$

where $\frac{\gamma^{*} p}{p^{*}}=1+\frac{(p-1)}{p^{*}}>1$. The reasoning above leads to the following enhanced stability result. 
Theorem 4.1 Assume $p \geq 2$ and $f \equiv 0$. The stationary solution $u^{*}$ is an $L^{p^{*}}$-asymptotically stable equilibrium with a region of attraction $\mathcal{R}_{u^{*}} \supseteq \mathcal{B}_{0}$, i.e. $V^{*}(t)$ satisfies a Lyapunov inequality of the type

$$
\dot{V}^{*}(t) \leq-c\left[V^{*}(t)\right]^{\frac{\gamma^{*} p}{p^{*}}}, \quad c=p^{* \frac{\gamma^{*} p}{p^{*}}}\left(\frac{S_{p}}{\gamma^{*}}\right)^{p}|\Omega|^{1-\gamma^{*}}>0
$$

for any initial condition $u_{0}(x) \in \mathcal{B}_{0}$.

Proof. The proof follows immediately by merging conditions (10)-(11), and observing that the integral in the right-hand side of (11) equals $p^{*} V^{*}(t)$.

\subsection{Lower-order terms: exponential stability}

Let us consider now a variation of the original differential problem, namely

$$
\left\{\begin{array}{l}
u_{t}=\operatorname{div}\left(|\nabla u|^{p-2} \nabla u\right)+g(u)+f \\
u(t, x)=0 \quad t \in[0, \infty), x \in \partial \Omega \\
u(0, x)=u_{0}(x) \quad x \in \Omega
\end{array}\right.
$$

Assumption 4.1 The lower-order term $g(\cdot)$ verifies suitable growth conditions:

$$
\begin{gathered}
-\bar{\lambda}|w|^{2}-\bar{k}(w) \leq g(w) w \leq-\underline{\lambda}|w|^{2}-\underline{k}(w) \quad \forall w \in \mathbb{R} \\
(g(w)-g(v))(w-v) \leq-\alpha|w-v|^{2}-\beta(w, v) \quad \forall w, v \in \mathbb{R}
\end{gathered}
$$

where $\bar{\lambda} \geq \underline{\lambda} \geq 0, \alpha>0$ and $\bar{k}(\cdot), \underline{k}(\cdot), \beta(\cdot, \cdot) \geq 0$ are smooth non-negative functions with $\bar{k}(0)=\underline{k}(0)=0$.

Remark 4.1 We notice that a simple and natural example of function $g(\cdot)$ fulfilling the latter conditions is

$$
g(w)=-\lambda w-\mu|w|^{p-2} w, \quad \mu \geq 0 .
$$

As mentioned, to simplify the presentation, it will be assumed $f \equiv 0$. We will prove that the presence of a lower-order term satisfying Assumption 4.1 with $\underline{\lambda}>0$ guarantees an improvement of the stability condition, i.e. it provides exponential stability of the equilibrium. To this end, let us consider once again the Lyapunov function candidate $V(t)$ defined in (5).

Theorem 4.2 Assume $p \geq 2, f \equiv 0$ and suppose that Assumption 4.1 is satisfied with $\underline{\lambda}>0$. The stationary solution $u^{*}$ of the problem (12) is an $L^{2}$ exponentially stable equilibrium with a region of attraction $\mathcal{R}_{u^{*}} \supseteq \mathcal{B}_{0}$, i.e. $V(t)$ satisfies a Lyapunov inequality of the type

$$
\dot{V}(t) \leq-2 \underline{\lambda} V(t)
$$

for any initial condition $u_{0}(x) \in \mathcal{B}_{0}$. 
Proof. Repeating the first part of the proof of Theorem 3.1 one gets

$$
\dot{V}(t) \leq-\int_{\Omega}|\nabla u|^{p} d x+\int_{\Omega} u g(u) d x,
$$

and hence Assumption yields

$$
\begin{aligned}
\dot{V}(t) & \leq-\int_{\Omega}|\nabla u|^{p} d x-\int_{\Omega}\left(\underline{\lambda}|u|^{2}+\underline{k}(u)\right) d x \\
& \leq-\underline{\lambda} \int_{\Omega}|u|^{2} d x=-2 \underline{\lambda} V(t),
\end{aligned}
$$

that is the desired condition. In particular, exponential stability is guaranteed thanks to

$$
V(t) \leq e^{-2 \underline{\lambda} t} V(0)=\frac{1}{2} e^{-2 \underline{\lambda} t}\left\|u_{0}\right\|_{2}^{2}
$$

\subsection{Degenerate parabolic equations in general form}

We present here a wider class of equations for which the stability theory developed so far is still valid. In this regard, let us emphasize that rather than the explicit form of the $p$-Laplace operator, its growth conditions have been used to attain the desired estimates.

Consider the following differential problem

$$
\left\{\begin{array}{l}
u_{t}=\operatorname{div}(a(x, t, \nabla u))+g(x, t, u)+f \\
u(t, x)=0 \quad t \in[0, \infty), x \in \partial \Omega \\
u(0, x)=u_{0}(x) \quad x \in \Omega
\end{array}\right.
$$

and, following the setting given in [17], let us make some preliminary assumptions.

C1) The mapping $(\xi, \sigma) \mapsto a(\xi, \sigma, z)$ is measurable for all $z \in \mathbb{R}^{n}$, and the mapping $z \mapsto a(\xi, \sigma, z)$ is continuous for a.e. $(\xi, \sigma) \in \mathbb{R}^{n} \times \mathbb{R}$.

C2) There exist constants $c_{2} \geq c_{1}>0$ with

$$
c_{1}|z|^{p} \leq a(\xi, \sigma, z)^{T} z \leq c_{2}|z|^{p}
$$

for all $z \in \mathbb{R}^{n}$ and for a.e. $(\xi, \sigma) \in \mathbb{R}^{n} \times \mathbb{R}$.

C3) For a.e. $(\xi, \sigma) \in \mathbb{R}^{n} \times \mathbb{R}$

$$
(a(\xi, \sigma, z)-a(\xi, \sigma, \zeta))^{T}(z-\zeta)>0
$$

for all $z, \zeta \in \mathbb{R}^{n}$ with $z \neq \zeta$. 
C3') For a.e. $(\xi, \sigma) \in \mathbb{R}^{n} \times \mathbb{R}$

$$
(a(\xi, \sigma, z)-a(\xi, \sigma, \zeta))^{T}(z-\zeta) \geq \theta|z-\zeta|^{p}, \theta>0
$$

for all $z, \zeta \in \mathbb{R}^{n}$.

C4) The lower-order term $g(\xi, \sigma, w)$ fulfills Assumption 4.1 uniformly in $(\xi, \sigma)$.

The following general result can be then established with the same technique that has been used to prove Theorem 3.1 and Theorem 3.2.

Proposition 4.1 Consider the degenerate parabolic problem (13) and assume that conditions $C 1, C 2$ and $C 4$ hold true with $p \geq 2$. The achievable ${ }^{3}$ stability results are summarized in Table I.

Table 1: Stability results for the degenerate parabolic equation

\begin{tabular}{cccc}
\hline Type of stability & $\mathrm{C} 3+\{\underline{\lambda}>0\}$ & $\mathrm{C} 3+\{\underline{\lambda}=0\}$ & $\mathrm{C} 3^{\prime}$ \\
\hline$L^{2}$-asymptotic & $\sqrt{ }$ & - & $\sqrt{ }$ \\
\hline$L^{p^{*}}$-asymptotic & $\sqrt{ }$ & - & $\sqrt{ }$ \\
\hline$L^{2}$-exponential & $\sqrt{ }$ & - & - \\
\hline
\end{tabular}

\section{Distributed control}

Having established the stability of the unforced equation, we are interested in considering a control problem where, by means of a distributed input, the task is to make the solution of the equation as close as possible to a desired target profile. In other words, we will focus on the controlled equation

$$
\left\{\begin{array}{l}
u_{t}=\operatorname{div}\left(|\nabla u|^{p-2} \nabla u\right)+v(t, x) \\
u(t, x)=\gamma(t, x) \quad t \in[0, \infty), x \in \partial \Omega \\
u(0, x)=u_{0}(x) \quad x \in \Omega
\end{array}\right.
$$

where the distributed input $v(t, x)$ has to be designed on order to guarantee $u(t, x) \approx u_{d}(t, x)$, for an assigned reference function $u_{d}(t, x)$. Note that even though no external source $f(t, x)$ is considered here, this might be easily handled by introducing a compensating term in the control input. Two different approaches will be presented, namely finite-time reference tracking and quadratic dynamic optimization.

\footnotetext{
${ }^{3}$ We notice that, when $\underline{\lambda}=0$, the condition C3 is sufficient to guarantee asymptotic stability of the equilibrium only in the homogeneous case $f \equiv 0$
} 


\subsection{Finite-time stabilization}

Introducing the tracking error

$$
\varepsilon(t, x):=u(t, x)-u_{d}(t, x),
$$

the aim of finite-time stabilization is making $\epsilon(\cdot, x)$ converge to zero in a finite time. We first observe that, in order for such objective to be feasible, the boundary conditions of the solution have to be consistent with those of the reference, this motivating the following assumption.

Assumption 5.1 The reference $u_{d}(t, \cdot) \in W^{1, p}(\Omega)$ is such that

$$
u_{d}(t, x)=\gamma(t, x) \forall x \in \partial \Omega \forall t \in[0,+\infty)
$$

and therefore the tracking error $\varepsilon(t, x)$ satisfies homogeneous Dirichlet conditions

$$
\varepsilon(t, \cdot) \in W_{0}^{1, p}(\Omega) \quad \forall t \in[0,+\infty)
$$

With the latter condition in mind, we propose a control input of the form

$$
v(t, x)=\partial_{t} u_{d}-\Delta_{p} u_{d}-\Pi(\varepsilon),
$$

consisting of a feed-forward term and a feedback term, where the operator $\Pi(\cdot)$ is designed as the finite-time stabilizer

$$
\Pi(\varepsilon)=-\kappa \frac{\operatorname{sign}(\varepsilon(t, x))|\varepsilon(t, x)|^{\beta}}{\|\varepsilon(t, \cdot)\|_{p^{*}}}
$$

with $\kappa>0$ and $\beta \in(1,2)$. By this choice, the error dynamics becomes

$$
\varepsilon_{t}=\Delta_{p} u-\Delta_{p} u_{d}+\Pi(\varepsilon)
$$

Remark 5.1 It is worth noticing that for any $2 \leq p<N$, there exists $\bar{\beta} \in(1,2)$ such that the operator $\Pi: L^{p^{*}} \rightarrow L^{p^{\prime}}$ is continuous for any $\beta \in(1, \bar{\beta})$, with

$$
\|\Pi(w)\|_{p^{\prime}} \leq c_{0}\left(\|w\|_{p^{*}}\right)^{\beta-1} \quad c_{0}>0,
$$

and thus equation (17) is well posed. In the opposite case $p \geq N$ (or, for the sake of simplicity in the general case $2 \leq p<+\infty)$, the easiest operation is adopting a standard $L^{2}$-version of this finite-time controller, namely replacing the term at the denominator with $\|\epsilon(t, \cdot)\|_{2}$.

Theorem 5.1 Let $2 \leq p<N$. Consider the tracking error $\varepsilon(t, x)=u(t, x)-$ $u_{d}(t, x)$ and the control input (15)-(16). There exists a region $\mathcal{R} \supseteq \mathcal{B}_{0}$ such that, for any initial condition $\epsilon_{0}(x):=u_{0}(x)-u_{d}(0, x) \in \mathcal{R}$, there exists a finite number $\tau\left(\epsilon_{0}(\cdot)\right)$ with

$$
\begin{gathered}
\lim _{t \rightarrow \tau\left(\epsilon_{0}(\cdot)\right)^{+}}\|\epsilon(t, \cdot)\|_{p^{*}}=0 \\
\|\epsilon(t, \cdot)\|_{p^{*}}=0 \quad \forall t \geq \tau\left(\epsilon_{0}(\cdot)\right)
\end{gathered}
$$


Proof. Let us consider the Lyapunov function candidate

$$
V^{*}(t)=\frac{1}{p^{*}} \int_{\Omega}|\varepsilon(t, x)|^{p^{*}} d x
$$

Computing the derivative along the solution of (17) yields

$$
\begin{aligned}
\dot{V}^{*}(t) & =\int_{\Omega}|\varepsilon(t, x)|^{p^{*}-2} \varepsilon(t, x) \cdot \varepsilon_{t}(t, x) d x \\
& =\int_{\Omega}|\varepsilon(t, x)|^{p^{*}-2} \varepsilon(t, x) \cdot\left(\Delta_{p} u-\Delta_{p} u_{d}\right) d x \\
& +\int_{\Omega}|\varepsilon(t, x)|^{p^{*}-2} \varepsilon(t, x) \cdot \Pi(\varepsilon) d x \\
& =-\int_{\Omega} \nabla\left(|\varepsilon(t, x)|^{p^{*}-2} \varepsilon(t, x)\right)^{T}\left(|\nabla u|^{p-2} \nabla u-\left|\nabla u_{d}\right|^{p-2} \nabla u_{d}\right) d x \\
& -\kappa \int_{\Omega} \frac{|\varepsilon(t, x)|^{p^{*}+\beta-1}}{\|\varepsilon(t, \cdot)\|_{p^{*}}} d x \\
& \leq-\int_{\Omega}|\epsilon(t, x)|^{p^{*}-1}|\nabla \varepsilon(t, x)|^{p} d x-\kappa \int_{\Omega} \frac{|\varepsilon(t, x)|^{p^{*}+\beta-1}}{\|\varepsilon(t, \cdot)\|_{p^{*}}} d x
\end{aligned}
$$

Let us focus on the last term and apply Jensen's inequality. One gets

$$
\dot{V}^{*}(t) \leq-\frac{\kappa}{|\Omega|^{\frac{\beta-1}{p^{*}}}}\left(\int_{\Omega}|\varepsilon(t, x)|^{p^{*}} d x\right)^{\frac{p^{*}+\beta-2}{p^{*}}}=-\tilde{\kappa}[V(t)]^{\frac{p^{*}+\beta-2}{p^{*}}}
$$

with $\tilde{\kappa}:=\frac{\kappa p^{*} \frac{p^{*}+\beta-2}{p^{*}}}{|\Omega|^{\frac{\beta-1}{p^{*}}}}$ and where, by construction

$$
\frac{p^{*}+\beta-2}{p^{*}} \in(0,1) .
$$

By a standard Lyapunov argument, the finite-time convergence is established, with

$$
V^{*}(t) \leq \max \left\{0,\left(\frac{\beta-2}{p^{*}}\left(\tilde{k} t+\frac{p^{*} V(0)^{\frac{2-\beta}{p^{*}}}}{\beta-2}\right)\right)^{\frac{p^{*}}{2-\beta}}\right\}
$$

In particular, given the initial error $\varepsilon_{0}(x):=u_{0}(x)-u_{d}(0, x)$, the settling time $\tau\left(\varepsilon_{0}(\cdot)\right)$ satisfies the upper bound

$$
\tau\left(\epsilon_{0}(\cdot)\right) \leq \frac{p^{* \frac{p^{*}+\beta-2}{p^{*}}}\left\|\varepsilon_{0}\right\|_{p^{*}}^{\frac{2-\beta}{p^{*}}}}{\tilde{k}(2-\beta)}
$$

and this concludes the proof. 


\subsection{Quadratic optimal control}

Let us now consider a finite time horizon $[0, T]$ and introduce a quadratic cost functional to be minimized

$$
\begin{aligned}
J(v) & =\frac{1}{2} \int_{0}^{T}\left(\int_{\Omega}\left|u(t, x)-u_{d}(t, x)\right|^{2} d x+\Gamma \int_{\Omega}|v(t, x)|^{2} d x\right) d t \\
& +\frac{K}{2} \int_{\Omega}\left|u(T, x)-u_{d}(T, x)\right|^{2} d x, \quad \Gamma, K>0 .
\end{aligned}
$$

where the first two terms are the running cost and the last term is the terminal cost. Furthermore, let us introduce the operator $\Delta_{p}^{\ddagger}: W^{1, p}(\Omega) \times W^{1, p}(\Omega) \rightarrow$ $W^{-1, p^{\prime}}(\Omega)$ defined by

$$
\Delta_{p}^{\ddagger}\left(u_{1}, u_{2}\right)=\operatorname{div}\left(\left|\nabla u_{2}\right|^{p-2} \nabla u_{1}\right)+(p-2) \operatorname{div}\left(\left(\nabla u_{1}^{T} \nabla u_{2}\right)\left|\nabla u_{2}\right|^{p-4} \nabla u_{2}\right),
$$

which can be regarded as a pseudo-adjoint operator of the $p$-Laplacian $\Delta_{p}$. The latter has been formally obtained by computing the Fréchet derivative of the duality product $\left\langle u_{1}, \Delta_{p} u_{2}\right\rangle$. Rewriting the cost function as

$$
J(v)=\int_{0}^{T} \mathcal{L}(t, u, v) d t+\mathcal{K}(T, u(T, \cdot))
$$

with suitable definitions of the running $\operatorname{cost} \mathcal{L}(\cdot, \cdot, \cdot)$ and the terminal cost $\mathcal{K}(\cdot, \cdot)$, we can introduce the adjoint equation for the co-state function $z \in V^{p}(0, T ; \Omega)$

$$
\begin{aligned}
z_{t} & =-\Delta_{p}^{\ddagger}(z, u)-\frac{\partial \mathcal{L}(t, u, v)}{\partial u} \\
& =-\Delta_{p}^{\ddagger}(z, u)-\left(u(t, x)-u_{d}(t, x)\right) \\
z(T, x) & =-\frac{\partial \mathcal{K}(T, u(T, x))}{\partial u}=-K\left(u(T, x)-u_{d}(T, x)\right)
\end{aligned}
$$

Theorem 5.2 Let $\left(u^{\star}(t, x), z^{\star}(t, x)\right)$ a solution of the system of coupled nonlinear equations (14)-(19) in $Q_{T}$, where the input $v(t, x)$ is assigned by the co-state feedback

$$
v^{\star}(t, x)=-\frac{z^{\star}(t, x)}{\Gamma} .
$$

Then $v^{\star}(t, x)$ is the optimal control with respect to the cost functional (18), i.e. $J\left(v^{\star}\right)=\min _{v \in L^{p^{\prime}}\left(Q_{T}\right)} J(v)$.

Proof. Let $v^{\star}(t, x)$ be the optimal control for our problem, and denote by $u^{\star}(t, x)$ the corresponding optimal trajectory. Furthermore, let us consider the perturbed input $v^{\star}(t, x)+\lambda \phi(t, x)$ where $\lambda \in \mathbb{R}^{+}$and $\phi(t, x) \in L^{p^{\prime}}\left(Q_{T}\right)$ is arbitrary. Accordingly, we denote by $u_{\lambda}(t, x)$ the associated trajectory. The difference function $\eta=u_{\lambda}-u^{\star}$ satisfies the differential condition

$$
\begin{aligned}
& \eta_{t}=\Delta_{p} u_{\lambda}-\Delta_{p} u^{\star}+\lambda \phi(t, x) \\
& \eta(t, x)=0 \quad \forall x \in \partial \Omega, \forall t \in[0, T] \\
& \eta(0, x)=0 \quad \forall x \in \Omega
\end{aligned}
$$


Multiplication by $\eta$, integration on $Q_{T}$ and property (4) yield

$$
\frac{1}{2} \int_{\Omega}|\eta(T, x)|^{2} d x+\frac{1}{2^{p-1}} \int_{0}^{T} \int_{\Omega}|\nabla \eta|^{p} d x d t \leq \lambda \int_{0}^{T} \int_{\Omega} \phi \eta d x d t
$$

which can be arranged as

$$
\int_{0}^{T} \int_{\Omega}|\nabla \eta|^{p} d x d t \leq c_{0} \lambda^{p}\|\phi\|_{p^{\prime}}^{p^{\prime}}
$$

using Young and Poincaré inequalities, for a suitable constant $c_{0}>0$. In particular, the latter condition implies that $\eta / \lambda$ is bounded in $V^{p}(0, T ; \Omega)$ and thus there exists (up to a subsequence) a weak limit $\eta_{\bullet} \in V^{p}(0, T ; \Omega)$ such that $\forall t \in[0, T]$

$$
\begin{aligned}
\lim _{\lambda \rightarrow 0}\left\langle\frac{\eta(t, \cdot)}{\lambda}, \varphi(\cdot)\right\rangle & =\left\langle\eta_{\bullet}(t, \cdot), \varphi(\cdot)\right\rangle \quad \forall \varphi \in W^{-1, p^{\prime}}(\Omega) \\
\lim _{\lambda \rightarrow 0} \frac{d}{d t}\left\langle\frac{\eta(t, \cdot)}{\lambda}, \vartheta(\cdot)\right\rangle & =\frac{d}{d t}\left\langle\eta_{\bullet}(t, \cdot), \vartheta(\cdot)\right\rangle \quad \forall \vartheta \in W_{0}^{1, p}(\Omega),
\end{aligned}
$$

Computing the Gateaux derivative of $J\left(v^{\star}\right)$ along the direction $\phi$ yields

$$
\lim _{\lambda \rightarrow 0} \frac{J\left(v^{\star}+\lambda \phi\right)-J\left(v^{\star}\right)}{\lambda}=\delta\left[J\left(v^{\star}\right)\right] \phi .
$$

Due to the optimality assumption, the first variation must satisfy

$$
\delta\left[J\left(v^{\star}\right)\right]=0 .
$$

The following technical result will be useful, and its proof is given in Appendix C.

Lemma 5.1 Let the functions $w_{1}(t, x), w_{2}(t, x) \in V^{p}(0, T ; \Omega)$ and $\psi \in L^{p^{\prime}}\left(Q_{T}\right)$ be such that

$$
\begin{aligned}
& \partial_{t}\left(w_{1}-w_{2}\right)=\Delta_{p} w_{1}-\Delta_{p} w_{2}+\psi \\
& w_{1}(t, x)=w_{2}(t, x) \quad \forall x \in \partial \Omega, \forall t \in[0, T] \\
& w_{1}(0, x)=w_{2}(0, x) \quad \forall x \in \Omega
\end{aligned}
$$

Furthermore, given the functions $\xi \in L^{p^{\prime}}\left(Q_{T}\right)$ and $z_{T} \in L^{p}(\Omega)$, let us consider the adjoint equation

$$
\begin{aligned}
& \partial_{t} z=-\Delta_{p}^{\ddagger}\left(z, w_{2}\right)-\xi \\
& z(T, x)=z_{T}(x) \quad \forall x \in \Omega
\end{aligned}
$$

Then the following duality condition holds true

$$
\begin{aligned}
& \int_{Q_{T}}\left(w_{1}-w_{2}\right) \xi d x d t+\int_{\Omega}\left(w_{1}(T)-w_{2}(T)\right) z_{T} d x \\
= & \int_{Q_{T}} z \psi d x d t+o\left(\left\|w_{1}-w_{2}\right\|_{p}\right)
\end{aligned}
$$


By a direct computation of the increment $J\left(v^{\star}+\lambda \phi\right)-J\left(v^{\star}\right)$ one gets

$$
\begin{aligned}
& J\left(v^{\star}+\lambda \phi\right)-J\left(v^{\star}\right) \\
= & \frac{1}{2} \int_{0}^{T} \int_{\Omega}\left(u_{\lambda}+u^{\star}-2 u_{d}\right)\left(u_{\lambda}-u^{*}\right) d x d t \\
+ & \frac{\Gamma}{2} \int_{0}^{T} \int_{\Omega}\left(2 \lambda \phi v^{\star}+\lambda^{2} \phi^{2}\right) d x d t \\
+ & \frac{K}{2} \int_{\Omega}\left(u_{\lambda}(T)+u^{\star}(T)-2 u_{d}(T)\right)\left(u_{\lambda}(T)-u^{*}(T)\right) d x
\end{aligned}
$$

Now applying Lemma 5.1 with $w_{1}=u_{\lambda}, w_{2}=u^{\star}, \psi=\lambda \phi, \xi=\left(u_{\lambda}+u^{\star}-2 u_{d}\right) / 2$ and terminal condition $z_{\lambda, T}=-\frac{K}{2}\left(u_{\lambda}(T)+u^{\star}(T)-2 u_{d}(T)\right)$, one gets

$$
\begin{aligned}
& J\left(v^{\star}+\lambda \phi\right)-J\left(v^{\star}\right) \\
= & \int_{0}^{T} \int_{\Omega} \lambda z_{\lambda} \phi d x d t+\frac{\Gamma}{2} \int_{0}^{T} \int_{\Omega}\left(2 \lambda \phi v^{\star}+\lambda^{2} \phi^{2}\right) d x d t+o(\lambda)
\end{aligned}
$$

where $z_{\lambda}$ stands for the solution of the adjoint equation for such choice of parameters. Dividing by $\lambda$ and taking the limit as $\lambda$ tends to zero one gets

$$
\delta\left[J\left(v^{\star}\right)\right] \phi=\int_{0}^{T} \int_{\Omega}\left(z^{\star}+\Gamma v^{\star}\right) \phi d x d t,
$$

where the identity $\frac{u_{\lambda}-u^{\star}}{\lambda}=u^{\star}+\lambda \eta_{\bullet}+o(\lambda)$ has been used and where $z^{\star} \in$ $W_{0}^{1, p}(\Omega)$ is defined as the solution of the adjoint equation in the limit case $\lambda \rightarrow 0^{+}$, i.e.

$$
\begin{aligned}
z_{t}^{\star} & =-\Delta_{p}^{\ddagger}\left(z^{\star}, u^{\star}\right)-\left(u^{\star}(t, x)-u_{d}(t, x)\right) \\
z^{\star}(T, x) & =-K\left(u^{\star}(T, x)-u_{d}(T, x)\right)
\end{aligned}
$$

Comparing (21) with condition (20), we can deduce the desired formula

$$
v^{\star}(t, x)=-\frac{z^{\star}(t, x)}{\Gamma}
$$

for the optimal control.

Remark 5.2 It is interesting noticing that the same result can be formally obtained by considering the Hamiltonian

$$
\mathcal{H}(t, u, v, z):=\left\langle z, \Delta_{p} u+v\right\rangle-\mathcal{L}(t, u, v)
$$

and applying an infinite-dimensional version of the Pontryagin maximum principle. Indeed, the optimal control $v^{\star}$ maximizes the Hamiltonian, and the optimal pair of state $u^{\star}(t, x)$ and costate $z^{\star}(t, x)$ are related by the system of canonical equations

$$
\left\{\begin{array}{l}
u_{t}=\frac{\partial \mathcal{H}(t, u, v, z)}{\partial z} \\
z_{t}=-\frac{\partial \mathcal{H}(t, u, v, z)}{\partial u}
\end{array}\right.
$$


where the differentiation in the right-hand side is performed in the sense of the Fréchet derivative.

\section{$6 \quad$ Numerical simulations}

Let us illustrate the proposed stability results by means of a series of numerical examples corresponding to different scenarios.

- Example 1: parabolic p-Laplace equation with zero data, i.e. $f \equiv 0$.

- Example 2: parabolic p-Laplace equation with $f \equiv 0$ and a lower-order term

$$
g(u)=-2 u-3|u|^{p-2} u .
$$

- Example 3: finite-time tracking of the reference function

$$
u_{d}\left(t, x_{1}, x_{2}\right)=\sin (t) \arctan \left(x_{1}^{2}+x_{2}^{2}\right)
$$

In each simulated scenario the space dimension is $n=2$ and the exponent $p=3$. The low dimension $n=2$ has been chosen only for allowing to describe graphically the behavior of solutions. For representation purposes, the domain $\Omega$ has been assumed to be the square $[-4,4]^{2}$, even though the boundary is not smooth at the vertices, this slightly weakening the theoretical assumptions used to establish the formal results. The initial condition has been set as

$$
u_{0}(x)=\sin \left(\frac{\pi}{4} x_{1}\right)\left(e^{-64}-e^{-x_{2}^{2}}\right) .
$$

The behavior of the solution of Example 1 is depicted of Figures 1-2, corresponding to evaluation at $t=10$ and $t=1000$ respectively. The asymptotic convergence to the stationary null solution, though quiet slow, can be easily deduced from the plots.

On the other hand, as proved in Section 4.2, the presence of a lower-order term provides additional regularity and guarantees exponential stability. This fact is shown in Figures 3-4, where the solution of Example 2 evaluated at $t=5$ and $t=100$ is reported: as expected, the solution is characterized by a remarkably higher convergence rate.

As a further comparison, the time histories of the $L^{2}$ norm of the solution of the $p$-parabolic equation with and without lower-order term are depicted in Figure 5.

Figure 6 illustrates the initial tracking error $\varepsilon_{0}(x)=u_{0}(x)-u_{d}(0, x)$ in Example 3 , where the initial condition has been selected as

$$
u_{0}(x)=\left(x_{1}^{2}-L^{2}\right)\left(x_{2}^{2}-L^{2}\right)
$$

while by construction $u_{d}(0, x) \equiv 0$. The finite-time controller (15)-(16) in its simplified $L^{2}$-version has been implemented (see Remark 5.1), and the finitetime convergence performance is well captured in Figures 7 and 8, where the persistency of peaks has to be ascribed to numerical errors. 


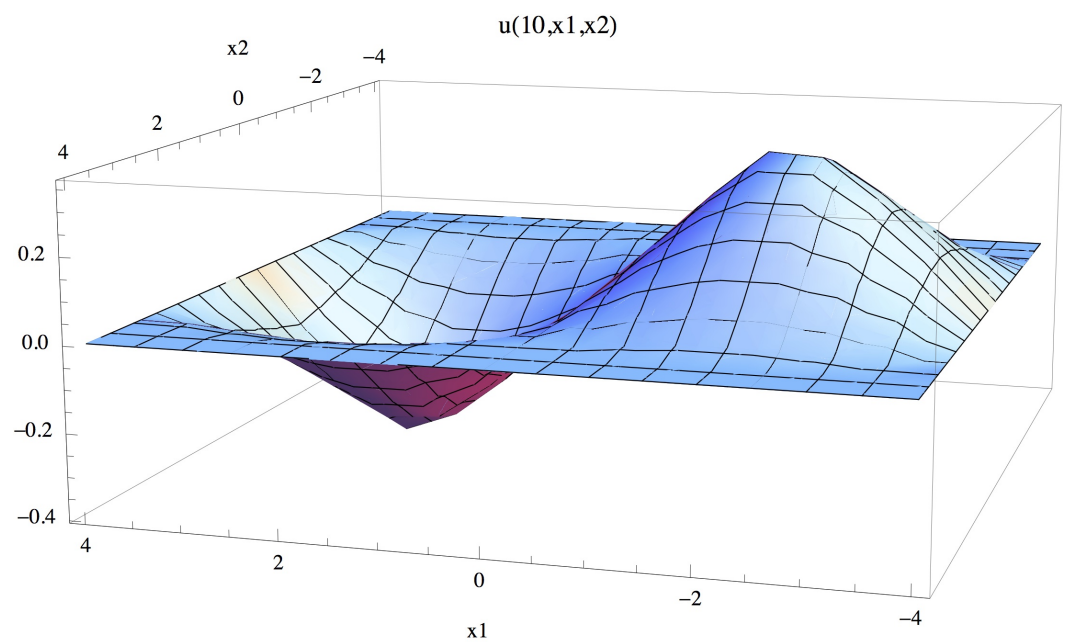

Figure 1: Example 1: solution $u\left(t, x_{1}, x_{2}\right)$ evaluated at $t=10$

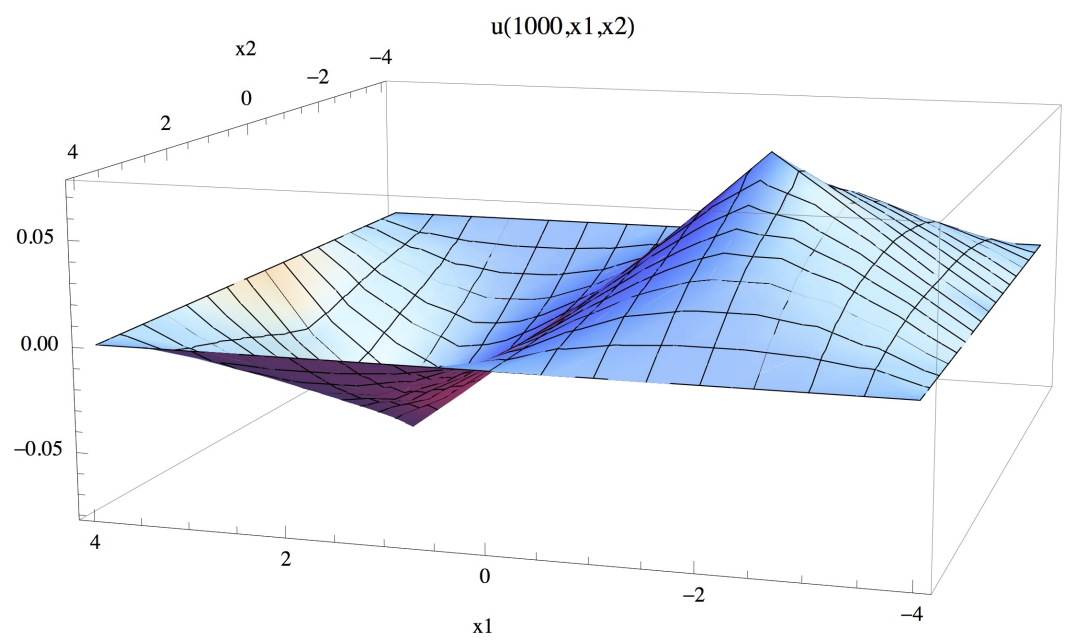

Figure 2: Example 1: solution $u\left(t, x_{1}, x_{2}\right)$ evaluated at $t=1000$

\section{References}

[1] Emilio Acerbi and Giuseppe Mingione. Gradient estimates for the p (x)-laplacian system. Journal für die reine und angewandte Mathematik, 2005(584):117-148, 2005.

[2] Robert A Adams and John JF Fournier. Sobolev spaces, volume 140. Academic press, 2003. 


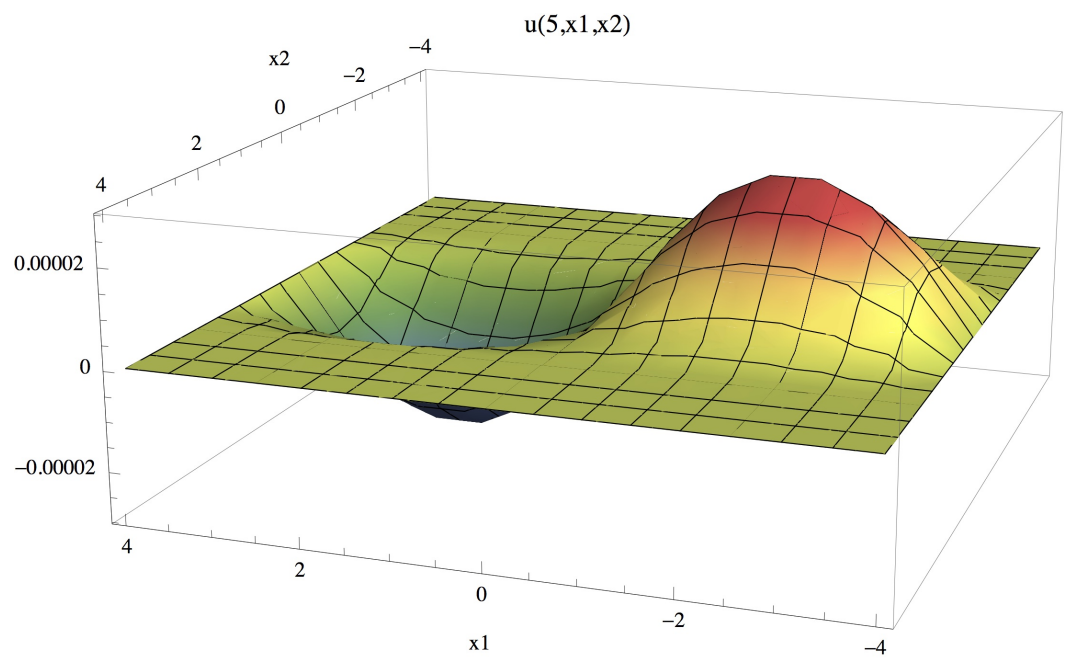

Figure 3: Example 2: solution $u\left(t, x_{1}, x_{2}\right)$ evaluated at $t=5$

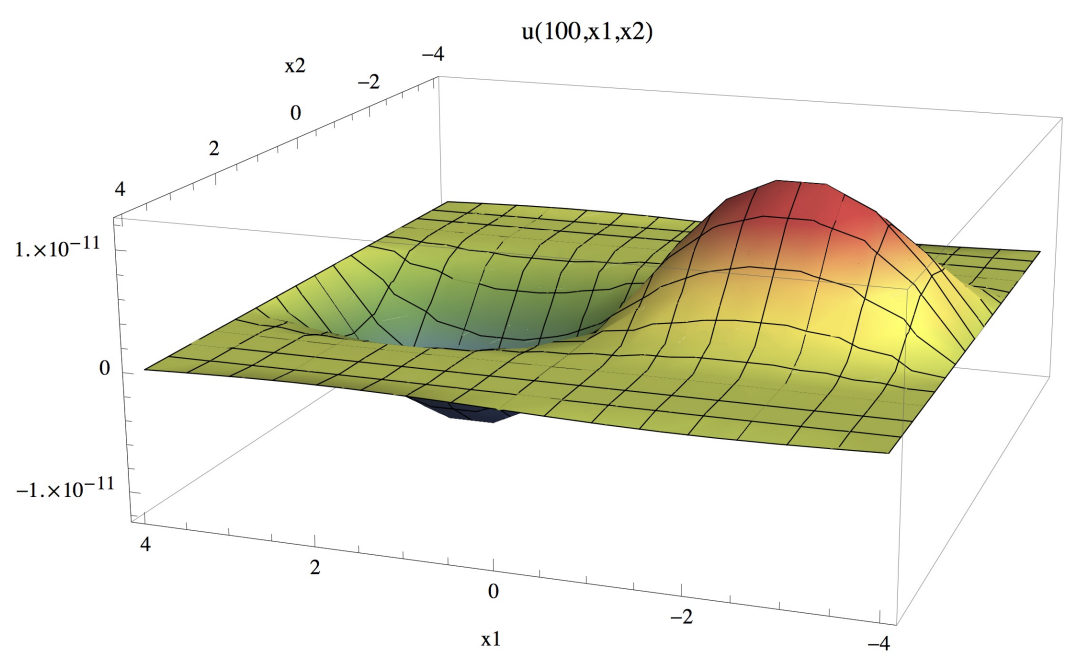

Figure 4: Example 2: solution $u\left(t, x_{1}, x_{2}\right)$ evaluated at $t=100$

[3] Henrik Anfinsen, Florent Di Meglio, and Ole Morten Aamo. Estimating the left boundary condition of coupled 1-d linear hyperbolic pdes from right boundary sensing. In Control Conference (ECC), 2016 European, pages 2179-2184, 2016.

[4] Guy Barles and Jérôme Busca. Existence and comparison results for fully nonlinear degenerate elliptic equations without zeroth-order term. Comm. in Partial Diff. Eq., 26(11-12):2323-2337, 2001. 


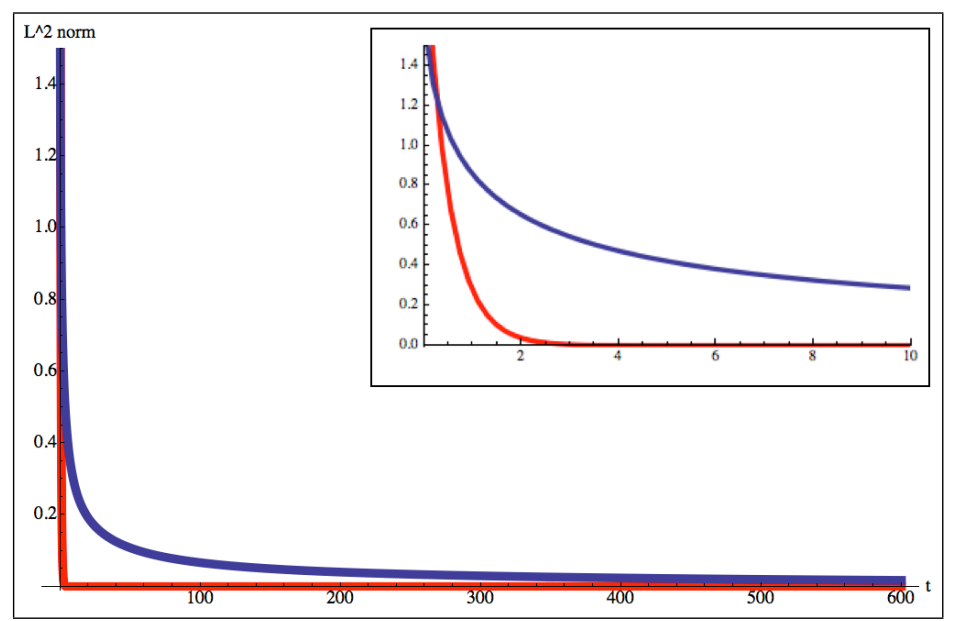

Figure 5: Time histories of the $L^{2}$ norm of solutions in Example 1 (blue) and Example 2 (red).

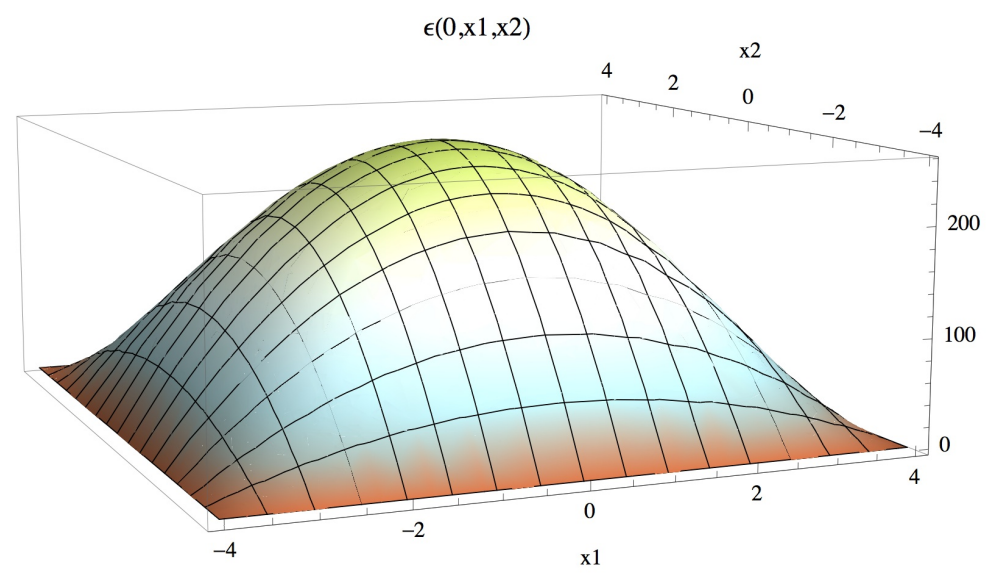

Figure 6: Example 3: initial error $u(0, x)-u_{d}(0, x)$

[5] Lucio Boccardo, Andrea Dall'Aglio, Thierry Gallouet, and Luigi Orsina. Existence and regularity results for some nonlinear parabolic equations. Adv. Math. Sci. Appl, 9(2):1017-1031, 1999.

[6] Richard G Casten and Charles J Holland. Stability properties of solutions to systems of reaction-diffusion equations. SIAM Journal on Applied Mathematics, 33(2):353-364, 1977.

[7] Felipe Castillo, Emmanuel Witrant, Christophe Prieur, and Luc Dugard. 


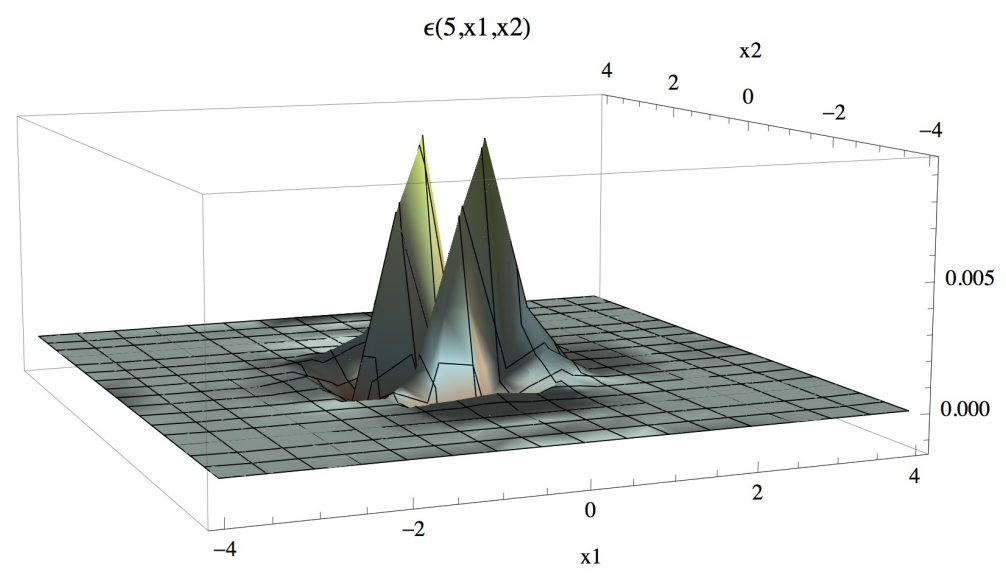

Figure 7: Example 3: tracking error $u(t, x)-u_{d}(t, x)$ evaluated at $t=5$

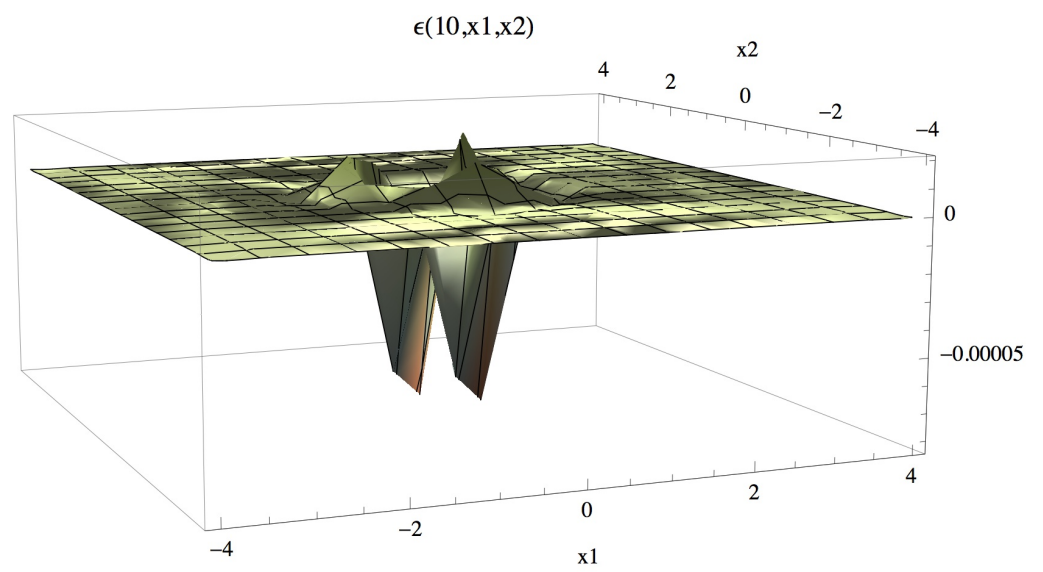

Figure 8: Example 3: tracking error $u(t, x)-u_{d}(t, x)$ evaluated at $t=10$

Dynamic boundary stabilization of linear and quasi-linear hyperbolic systems. In Decision and Control (CDC), 2012 IEEE 51st Annual Conference on, pages 2952-2957, 2012.

[8] Nathaniel Chafee. Asymptotic behavior for solutions of a one-dimensional parabolic equation with homogeneous neumann boundary conditions. Journal of Differential equations, 18(1):111-134, 1975.

[9] Silvia Cingolani and Marco Degiovanni. Nontrivial solutions for p-laplace equations with right-hand side having p-linear growth at infinity. Comm. in Partial Diff. Eq., 30(8):1191-1203, 2005. 
[10] Andrea Cristofaro. Robust tracking control for a class of perturbed and uncertain reaction-diffusion equations. IFAC Proceedings Volumes, 47(3):11375-11380, 2014.

[11] Andrea Cristofaro, Roberto Giambò and Fabio Giannoni. Lyapunov stability results for the parabolic p-Laplace equation. In European Control Conference, in press, 2018.

[12] Emmanuele DiBenedetto. Degenerate parabolic equations. Springer Science \& Business Media, 2012.

[13] Jens Frehse and Sebastian Schwarzacher. On regularity of the time derivative for degenerate parabolic systems. SIAM Journal on Mathematical Analysis, 47(5):3917-3943, 2015.

[14] E. Fridman and Y. Orlov. An LMI approach to $H_{\infty}$ boundary control of semilinear parabolic and hyperbolic systems. Automatica, 45:2060-2066, 2008.

[15] Juha Heinonen. Lectures on Lipschitz analysis. Technical report - University of Jyväskylä Department of Mathematics and Statistics, 100, 2005.

[16] Petri Juutinen. Principal eigenvalue of a very badly degenerate operator and applications. Journal of Diff. Equations, 236(2):532-550, 2007.

[17] Tero Kilpeläinen and Peter Lindqvist. On the Dirichlet boundary value problem for a degenerate parabolic equation. SIAM Journal on Mathematical Analysis, 27(3):661-683, 1996.

[18] M. Krstic and A. Smyshlyaev. Adaptive control of PDEs. Ann. Rev. Control, 32:149-160, 2008.

[19] An Lê. Eigenvalue problems for the p-Laplacian. Nonlinear Analysis: Theory, Methods \& Applications, 64(5):1057-1099, 2006.

[20] KiAhm Lee, Arshak Petrosyan, and Juan Luis Vázquez. Large-time geometric properties of solutions of the evolution p-laplacian equation. Journal of Differential Equations, 229(2):389-411, 2006.

[21] Tommaso Leonori and Francesco Petitta. Asymptotic behavior for solutions of parabolic equations with natural growth terms and irregular data. Asymptotic Analysis, 48(3):219-233, 2006.

[22] Peter Lindqvist. Notes on the p-Laplace equation. Norwegian University of Science and Technology, 2006.

[23] Peter Lindqvist. On the time derivative in an obstacle problem. Rev. Mat. Iberoam., 28:577-590, 2012.

[24] Juan J Manfredi. Isolated singularities of p-harmonic functions in the plane. SIAM Journal on Mathematical Analysis, 22(2):424-439, 1991. 
[25] Swann Marx, Eduardo Cerpa, Christophe Prieur, and Vincent Andrieu. Global stabilization of a korteweg-de vries equation with saturating distributed control. SIAM Journal on Control and Optimization, 55(3):14521480, 2017.

[26] V. Maz'ya. Sobolev Spaces. Springer, Berlin, 1984.

[27] Anthony N Michel, Ling Hou, and Derong Liu. Stability of dynamical systems. Springer, 2008.

[28] Pekka Neittaanmaki and Dan Tiba. Optimal control of nonlinear parabolic systems: theory: algorithms and applications, volume 179. CRC Press, 1994.

[29] A. Pisano, Y. Orlov, and E. Usai. Tracking control of the uncertain heat and wave equation via power-fractional and sliding-mode techniques. SIAM J. Control Optim., 49(2):363-382, 2011.

[30] Patrizia Pucci and Raffaella Servadei. Nonexistence for p-laplace equations with singular weights. Comm. Pure Appl. Anal, 9(5):1421-1438, 2010.

[31] H. Sira-Ramirez. Distributed sliding-mode control in systems described by quasilinear partial differential equations. Systems \& Control Letters, 13:177-181, 1989.

[32] Andrey Smyshlyaev and Miroslav Krstic. Backstepping observers for a class of parabolic pdes. Systems $\& 3$ Control Letters, 54(7):613-625, 2005.

[33] Fredi Tröltzsch. Optimal control of partial differential equations: theory, methods, and applications, volume 112. AMS, 2010.

[34] Mahamadi Warma. The fractional relative capacity and the fractional Laplacian with Neumann and Robin boundary conditions on open sets. Potential Analysis, 42(2):499-547, 2015.

\section{Appendix A - Some useful inequalities}

For the readers' convenience a collection of classical inequalities that have been used in the paper are reported.

Poincaré's inequality. Let $v(x) \in W_{0}^{1, p}(\Omega), p \geq 1$. There exists a positive constant $C_{p}>0$ such that

$$
\|v\|_{p} \leq C_{p}\|\nabla v\|_{p} .
$$

Jensen's inequality. Let $h(x) \in L^{1}(\Omega)$ be non-negative with $|\Omega|<\infty$, and consider a continuous, convex and non-negative function $\varphi: \mathbb{R}^{+} \rightarrow \mathbb{R}^{+}$. Then the following estimate holds:

$$
\varphi\left(\frac{1}{|\Omega|} \int_{\Omega} h(x) d x\right) \leq \frac{1}{|\Omega|} \int_{\Omega} \varphi(h(x)) d x .
$$


Gagliardo-Nirenberg-Sobolev inequality. Let $v(x) \in W_{0}^{1, p}(\Omega)$ and $1 \leq p<n$. There exists a positive constant $S_{p}>0$ such that

$$
\|v\|_{p^{*}} \leq S_{p}\|\nabla v\|_{p}
$$

where $p^{*}>p$, referred to as the Sobolev conjugate of $p$, is given by the relationship $1 / p^{*}=1 / p-1 / n$.

For a detailed discussion about these inequalities and their connections with more general properties of Sobolev spaces, one may refer to the classical textbooks $[2,26]$.

\section{Appendix B - Proof of Claim 3.1}

Let us demonstrate the claim. As proved in [13, Corollary 2.5], the solution $u(t, x)$ verifies

$$
u_{t}(t, x) \in W_{l o c}^{\alpha, 2}\left(Q_{T}\right) \quad \forall \alpha \in\left(0, \frac{1}{4}\right), \quad \forall T>0 .
$$

By the theory of fractional capacities of open sets, the function $u_{t}(t, x)$ admits a $\operatorname{cap}_{\alpha, 2}$-quasi continuous representative [34], i.e. $u_{t}(t, x)$ can be identified with a continuous function for any $(t, x) \in K \backslash \mathcal{E}$ with $\operatorname{cap}_{\alpha, 2}(\mathcal{E})=0$, where $K$ is an arbitrary compact subset $K \subset Q_{T}$. We recall that the capacity $\operatorname{cap}_{s, p}(\mathcal{A})$ of the open set $\mathcal{A}$ is defined as

$$
\operatorname{cap}_{s, p}(\mathcal{A})=\inf \left\{\|\omega\|_{W^{s, p}\left(\mathbb{R}^{n}\right)}^{p}: \begin{array}{l}
\omega \in W^{s, p}\left(\mathbb{R}^{n}\right), \\
\omega \geq 1 \text { a.e. in } \mathcal{A}
\end{array}\right\},
$$

and that the following implication holds

$$
\operatorname{cap}_{s, p}(\mathcal{E})=0 \Longrightarrow|\mathcal{E}|=0 \text {. }
$$

As a consequence, the function $u_{t}(t, x)$ admits an a.e.-quasi continuous representative.

Let us select now an arbitrary point of continuity $\left(t_{0}, x_{0}\right)$ for $u_{t}(t, x)$; by definition, the function $u_{t}(t, x)$ is then bounded in a neighbourhood $\mathcal{N}_{\left(t_{0}, x_{0}\right)}$ of $\left(t_{0}, x_{0}\right)$, and hence by Rademacher's theorem [15], $u(t, x)$ is differentiable a.e. in $\mathcal{N}_{\left(t_{0}, x_{0}\right)}$. Since $\left(t_{0}, x_{0}\right)$ is arbitrary, such a property holds a.e. in $Q_{T}$, and therefore the claim has been proved.

\section{Appendix C - Proof of Lemma 5.1}

Assume first $z_{T} \in W_{0}^{1, p}(\Omega)$; in this case, integration by parts yields the identity

$$
\begin{aligned}
\int_{0}^{T} \int_{\Omega} \partial_{t}\left(w_{1}-w_{2}\right) z d x d t & =-\int_{0}^{T} \int_{\Omega} \partial_{t} z\left(w_{1}-w_{2}\right) d x d t \\
& +\int_{\Omega}\left(w_{1}(T)-w_{2}(T)\right) z_{T} d x
\end{aligned}
$$


Now, using the equation satisfied by $\left(w_{1}-w_{2}\right)$ and $z$, we get

$$
\begin{aligned}
& \int_{0}^{T} \int_{\Omega}\left(\Delta_{p} w_{1}-\Delta_{p} w_{2}\right) z d x d t+\int_{0}^{T} \int_{\Omega} \psi z d x d t \\
= & \int_{0}^{T} \int_{\Omega} \Delta_{p}\left(w_{2}, z\right)\left(w_{1}-w_{2}\right) d x d t+\int_{0}^{T} \int_{\Omega} \xi\left(w_{1}-w_{2}\right) d x d t \\
+ & \int_{\Omega}\left(w_{1}(T)-w_{2}(T)\right) z_{T} d x
\end{aligned}
$$

Focusing on the first term, an applying the divergence lemma and taking the Taylor expansion centered in $\nabla w_{2}$, one has

$$
\begin{aligned}
& \int_{0}^{T} \int_{\Omega}\left(\Delta_{p} w_{1}-\Delta_{p} w_{2}\right) z d x d t \\
= & -\int_{0}^{T} \int_{\Omega}\left(\left|\nabla w_{1}\right|^{p-2} \nabla w_{1}-\left|\nabla w_{2}\right|^{p-2} \nabla w_{2}\right)^{T} \nabla z d x d t \\
= & \int_{0}^{T} \int_{\Omega} \Delta_{p}^{\ddagger}\left(w_{2}, z\right)\left(w_{1}-w_{2}\right) d x d t+\int_{0}^{T} \int_{\Omega} o\left(\nabla w_{1}-\nabla w_{2}\right) z d x d t .
\end{aligned}
$$

The duality formula has been then established for $z_{T} \in W_{0}^{1, p}(\Omega)$ : for the general case $z_{T} \in L^{p}(\Omega)$, it is sufficient to apply a density argument. 